\title{
High spatial resolution picosecond cathodoluminescence of InGaN quantum wells
}

\author{
S. Sonderegger, ${ }^{\text {a) }}$ E. Feltin, M. Merano, A. Crottini, J. F. Carlin, R. Sachot, B. Deveaud, \\ N. Grandjean, and J. D. Ganière \\ École Polytechnique Fédérale de Lausanne (EPFL), IPEQ, CH-1015 Lausanne, Switzerland
}

(Received 17 August 2006; accepted 13 October 2006; published online 5 December 2006)

\begin{abstract}
The authors have studied $\operatorname{In}_{x} \mathrm{Ga}_{1-x} \mathrm{~N} / \mathrm{GaN}(x \approx 15 \%)$ quantum wells $(\mathrm{QWs})$ using atomic force microscopy (AFM) and picosecond time resolved cathodoluminescence (pTRCL) measurements. They observed a contrast inversion between monochromatic CL maps corresponding to the high energy side $(3.13 \mathrm{eV})$ and the low energy side $(3.07 \mathrm{eV})$ of the QW luminescence peak. In perfect correlation with CL images, AFM images clearly show regions where the QW thickness almost decreases to zero. Pronounced spectral diffusion from high energy thinner regions to low energy thicker regions is observed in pTRCL, providing a possible explanation for the hindering of nonradiative recombination at dislocations. () 2006 American Institute of Physics.
\end{abstract}

[DOI: $10.1063 / 1.2397562]$

Since Nakamura et al. reported a candela-class blue light emitting diode in $1994,{ }^{1}$ GaN based nanostructures have been the subject of intense research efforts due to their increasing importance in today's optoelectronics. Because of the lack of a lattice matched substrate, InGaN based heterostructures suffer from a high threading dislocation density $\rho$ (up to $10^{10} \mathrm{~cm}^{-2}$ ). Because this high defect density should increase the nonradiative recombination of excited carriers, the high luminescence efficiency of InGaN based heterostructures was very surprising. In addition, InGaN exhibits large electric fields ${ }^{2}$ (some MV/cm) in the [0001] direction in the nanostructures which results in a redshift (quantum confined Stark effect) of the emission energies ${ }^{3,4}$ and in a separation of the electron and the hole also expected to decrease the radiative recombination probability. A commonly accepted explanation for the high luminescence efficiency of InGaN based nanostructures is provided by random localization of excitons on quantum dots or at compositional fluctuations prohibiting nonradiative recombination. ${ }^{5-7}$ However, the discussion was recently opened again by Hangleiter $e t$ $a l$., and an alternative mechanism was proposed. ${ }^{8}$ In this context, correlating morphology with temporally and spatially resolved luminescence signals is essential in the understanding of luminescence properties of $\mathrm{InGaN}_{\mathrm{Nased}}$ quantum wells (QWs). ${ }^{9-11}$ Cathodoluminescence (CL) techniques have proven to be very useful for studying various GaN based nanostructures. ${ }^{12,13}$ However, to get a complete picture of carrier recombination and diffusion processes, one needs a spectroscopic tool which yields high spatial and temporal resolutions at the same time.

In this letter we report on the spatial and temporal variation of the InGaN/GaN QWs emission by means of picosecond time resolved CL (pTRCL) measurements. Atomic force microscopy (AFM) is used to correlate optical measurements with the QW morphology. We have developed an original pTRCL setup using an ultrafast pulsed electron gun described elsewhere. ${ }^{14,15}$ Our setup reaches remarkable spatial and temporal resolutions of $50 \mathrm{~nm}$ and $10 \mathrm{ps}$, respectively. The samples were grown by metal-organic vapor phase epi-

${ }^{a)}$ Electronic mail: samuel.sonderegger@epfl.ch taxy (MOVPE) in an AIXTRON 200/4 RF-S reactor on 2 in. $c$-plane sapphire substrates at a temperature of $740{ }^{\circ} \mathrm{C}$. Two types of heterostructures were investigated. One kind of samples (A) is composed of single $\operatorname{In}_{x} \mathrm{Ga}_{1-x} \mathrm{~N} / \mathrm{GaN}$ QWs $(1.5 \mathrm{~nm}$ thick, $x \approx 15 \%)$ with or without any $\mathrm{GaN}$ cap layer deposited on standard $2 \mu \mathrm{m}$ thick GaN templates. Another sample (B) consists of two single $\operatorname{In}_{x} \mathrm{Ga}_{1-x} \mathrm{~N} / \mathrm{GaN}$ $(x \approx 15 \%)$ QWs grown on a nonintentionally doped epitaxial lateral overgrowth $\mathrm{GaN}$ template on sapphire $\left(\rho \approx 4 \times 10^{7} \mathrm{~cm}^{-2}\right)$. In this case the active structure is composed of a $3 \mathrm{~nm} \mathrm{InGaN} \mathrm{well,} \mathrm{a} 10 \mathrm{~nm} \mathrm{GaN}$ barrier, a thin $1.5 \mathrm{~nm} \mathrm{InGaN}$ well and a GaN cap layer $(10 \mathrm{~nm})$. The thinnest QW is located next to the surface and its luminescence peak is at higher energy $(3.10 \mathrm{eV})$ than the second well $(2.92 \mathrm{eV})$. The use of a larger well below the thin one improves the spatial resolution of CL measurements, as most of the carriers created within the large excitation volume are captured in the low energy well. Thus the CL resolution of the surface QW is given by the electron beam diameter $(\approx 50 \mathrm{~nm})$ plus some diffusion distance (see discussion below). Figure 1 shows AFM images of the sample surfaces (sample A) at different stages of the growth of an InGaN/GaN QW. A scan of the GaN template surface before
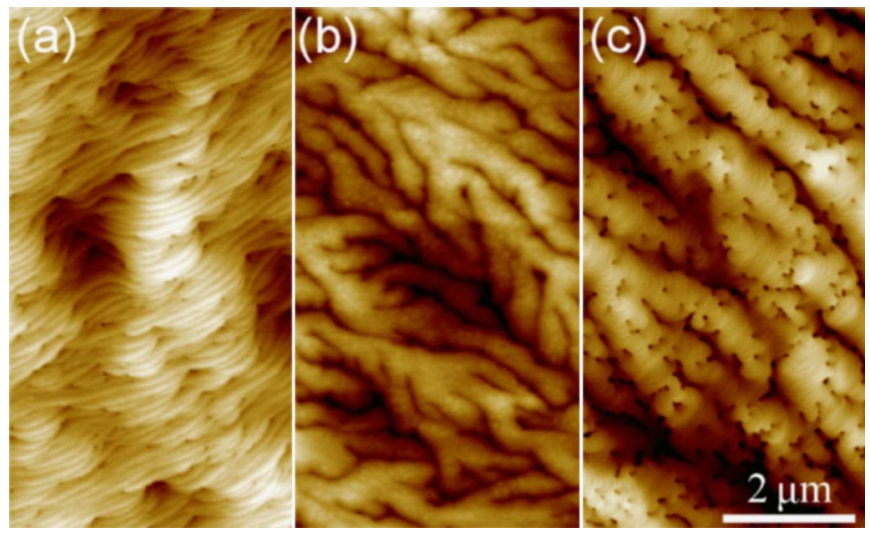

FIG. 1. (Color online) AFM images of the sample A surfaces at different growth stages: after (a) the GaN template growth, (b) after the growth of a $10 \mathrm{~nm} \mathrm{InGaN}$ layer, and (c) after the $10 \mathrm{~nm} \mathrm{GaN}$ cap layer deposition. 

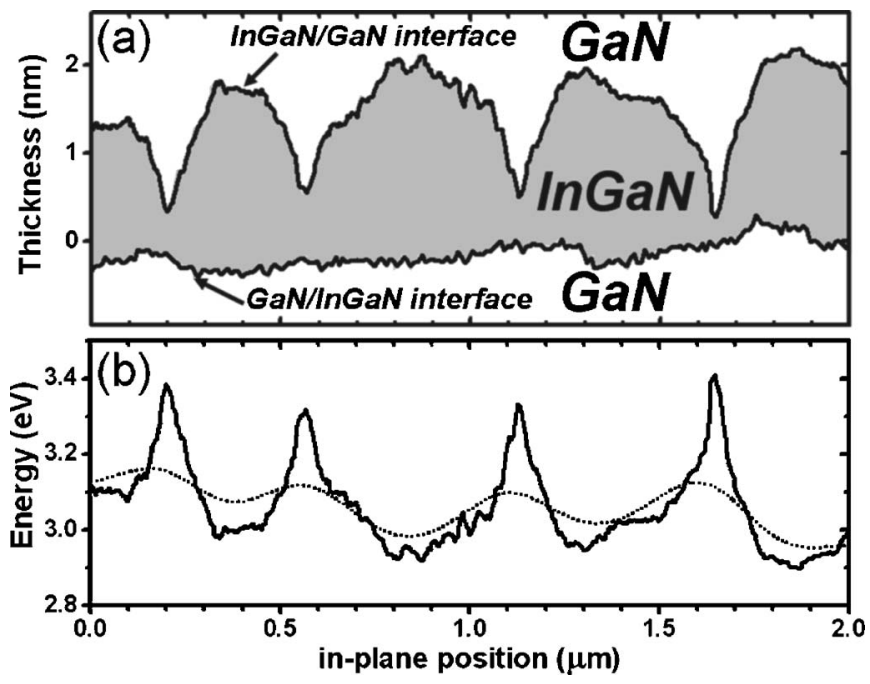

FIG. 2. (a) AFM profiles and (b) calculated energy profiles for the QW next to the surface. The dotted line represents an averaged energy profile. See text below for explanation.

the growth of the InGaN well is depicted in Fig. 1(a) $\left(\rho \approx 7 \times 10^{8} \mathrm{~cm}^{-2}\right)$. The surface has a low rms roughness of $0.25 \mathrm{~nm}$ on a $2 \times 2 \mu \mathrm{m}^{2}$ area. By comparison, the rms roughness of an InGaN layer deposited on this surface shown in Fig. 1(b) is highly increased $(0.65 \mathrm{~nm})$. The characteristic surface of the InGaN layer is composed of long valleys aligned in the $\langle 1-100\rangle$ directions. The depth of these valleys can reach approximately the nominal thickness of the well $(\approx 1.5 \mathrm{~nm})$. As a consequence, the thickness of the well is very inhomogeneous in the plane and is almost zero in the deep end of the valleys. The dislocations are no more visible in Fig. 1(b) due to the larger roughness of the surface. Finally, an image of the sample surface after deposition of a $10 \mathrm{~nm}$ thick GaN barrier is depicted in Fig. 1(c). The morphology is very similar to that observed on the InGaN well, which signifies that the well morphology is conserved after the growth of the GaN barrier. This particular morphology has already been observed for InGaN/GaN QWs grown by MOVPE. ${ }^{16}$ In Fig. 2(a) we present AFM section profiles corresponding to the AFM images in Figs. 1(a) and 1(b). Profiles are extracted along a direction perpendicular to the valleys. If we assume that the segregation and the diffusion of the indium atoms in the well are low during the growth, those sections correspond to the two InGaN/GaN interfaces of the QW. While one of the interfaces is very flat, the other interface exhibits a hilly profile due to the particular growth mode of InGaN. A characteristic distance between valleys is around $500 \mathrm{~nm}$. The maximum thickness of the well far from the valleys is nearly constant and corresponds approximately to the nominal thickness. However, the QW thickness varies very abruptly near the valleys where it reaches almost zero. The dark curve in Fig. 2(b) presents the energy variation of an $\mathrm{InGaN} / \mathrm{GaN} \mathrm{QW}$ calculated from the thickness variation in Fig. 2(a). This was determined using envelope function calculations for a homogeneous indium composition of $15 \%$ and an electric field $F=1800 \mathrm{kV} / \mathrm{cm}$. When taking into account the thickness profile in Fig. 2(a) the energy would vary over a range as large as $400 \mathrm{meV}$ over a distance of $\approx 500 \mathrm{~nm}$. Observed correlation between the QW morphology (see discussion below) and CL images indicate that energy shifts of the QW emission peak are mainly due to well Downloaded 06 Dec 2006 to 128.178.83.30. Redistribution subject
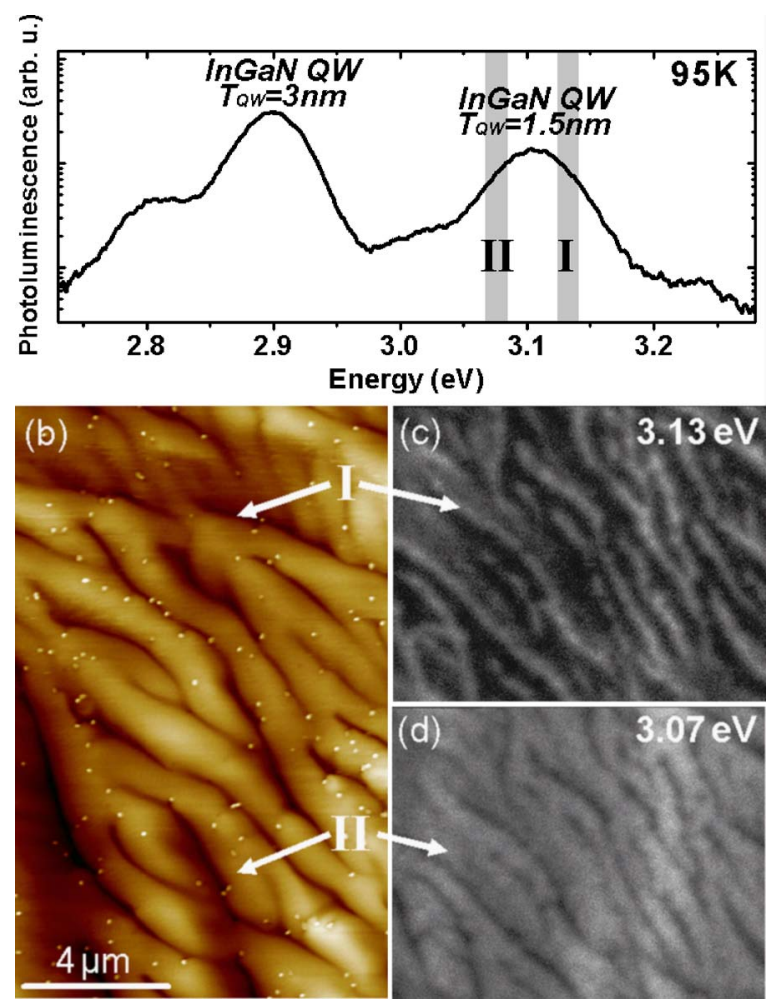

FIG. 3. (Color online) (a) CL spectra of sample B. Images (b) by AFM of the surface and by monochromatic CL (on a different location) at (c) $3.13 \mathrm{eV}$ and (d) $3.07 \mathrm{eV}$ of the thin InGaN/GaN QW of sample B.

thickness fluctuations. Of course, one cannot discard additional effects due to indium and strain gradients in the vicinity of the valleys. Further studies are thus required to give a more detailed picture.

A CL spectrum of sample B at $95 \mathrm{~K}$ is depicted in Fig. 3(a). The thin QW located near the surface is centered at $3.11 \mathrm{eV}$. Figures 3(c) and 3(d) show monochromatic CL maps of sample B also taken at $95 \mathrm{~K}$ corresponding to the high energy side $(3.13 \mathrm{eV})$ and the low energy side $(3.07 \mathrm{eV})$ of the QW luminescence peak in Fig. 3(a). Comparison between the thin line structure on the high energy CL map $(3.13 \mathrm{eV})$ and the valleys on the AFM image in Fig. 3(b) strongly suggests that these two structures coincide. This observation is further supported by the perfect contrast inversion between the high energy [Fig. 3(c)] and the low energy CL map [Fig. 3(d)]. In fact, Fig. 2(b) shows that a high energy emission is expected from the deep end of the valleys because the QW thickness is decreased there compared to the nominal thickness of $1.5 \mathrm{~nm}$. Local CL spectra from these valleys show a clear blueshift of the QW luminescence peak compared to the regions between the valleys [region II in Fig. 3(b)] where the QW has its nominal thickness. Moreover, the local CL spectra from the valleys have a full width at half maximum (FWHM) of $86 \mathrm{meV}$ compared to a FWHM of $57 \mathrm{meV}$ in the other zones. This can be understood by noting that the same number of excited carriers has access to more different energetic levels for recombination in the valleys due to the rapidly changing QW thickness. It is also important to remark that due to the fact that the valleys are well defined on the monochromatic CL images there is almost no carrier transport from outside the valleys into the valleys. Thus the thinner QW in the valleys acts as an energetic barrier hindering excited carriers from nonradito AIP license or copyright, see http://apl.aip.org/apl/copyright.jsp 


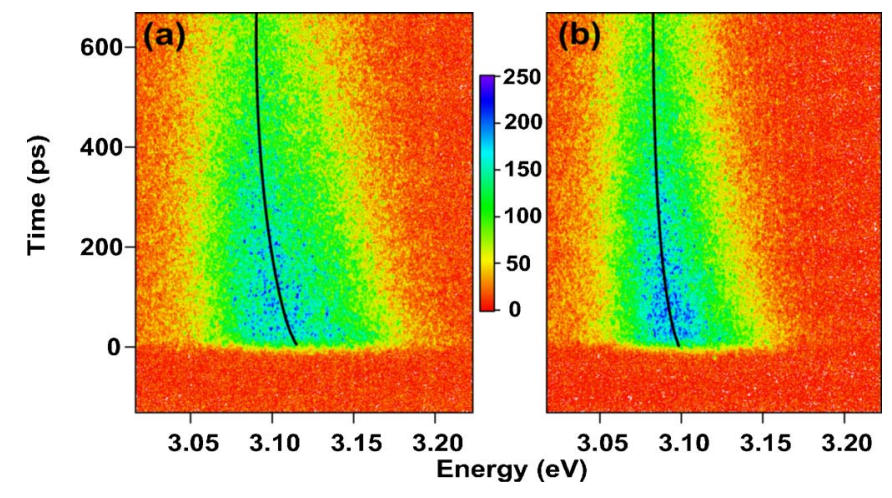

FIG. 4. (Color online) Streak camera traces (a) in the valley and (b) outside the valley region. Color coded intensities are in arbitrary units. Black lines are guides to the eye.

ative recombination on the dislocations in the valleys. A dynamic picture of the carrier diffusion is obtained by analyzing streak camera traces from inside and outside the valleys displayed on Figs. 4(a) and 4(b). A pronounced spectral diffusion $(\approx 80 \mathrm{meV})$ towards lower energy within $600 \mathrm{ps}$ after excitation is apparent. Considering that the excitation density is very low (taking into account the primary electron penetration depth for our acceleration voltage, we estimate an excited pair density of $10^{11} \mathrm{~cm}^{-2}$ ) this phenomenon cannot be due to the screening of the electric field in the well by excited carriers. In addition, the effect of the electric field on the luminescence energy (redshift) is very weak for such thin QWs $(<50 \mathrm{meV})$. As a consequence, this can be attributed to carrier diffusion processes from high to low energy regions. Assuming that the movement of the excited carriers is purely diffusive, using the measured average effective lifetime and a bipolar diffusion coefficient ${ }^{17}$ $D=2.1 \mathrm{~cm}^{2} \mathrm{~s}^{-1}$, we can estimate an excited carrier diffusion length of $L_{\text {diff }} \approx 100 \mathrm{~nm}$. Taking into account $L_{\text {diff }}$ and a beam radius of $50 \mathrm{~nm}$, we average the calculated energy profile in Fig. 2(b) (dotted line) using a Gaussian profile with a FWHM of $250 \mathrm{~nm}$ to obtain a more realistic energy variation for comparison with experimental data. The energy fluctuations are smoothed in this latter case but an amplitude of $E_{\text {diff }} \approx 100 \mathrm{meV}$ is still computed. The averaged energy variation [dotted line on figure 2(b)] over a distance $L_{\text {diff }}$ is in good agreement with the measured spectral diffusion.

In conclusion, we have correlated morphological information obtained by AFM with CL measurements of an InGaN/GaN QW. The InGaN well exhibits deep valleys where the QW thickness almost decreases to zero. Due to the thinner well in the valley, luminescence peaks are shifted to high energy in these regions as observed in local CL spectra. An exciton diffusion process is evidenced in $\mathrm{InGaN} / \mathrm{GaN}$ QWs by time resolved CL measurements. The energetic screening of dislocations in the valleys may provide an explanation of the high luminescence efficiency of $\mathrm{InGaN} / \mathrm{GaN}$ optoelectronic devices.

This work was carried out in the framework of the NCCR Quantum Photonics program of the Swiss National Science Foundation. One of the authors (N.G.) is indebted to the Sandoz Family Foundation for its support. The authors are grateful to M. Ilegems for continuous advice.

${ }^{1}$ S. Nakamura, T. Mukai, and M. Senoh, Appl. Phys. Lett. 64, 1687 (1994).

${ }^{2}$ F. Bernardini, V. Fiorentini, and D. Vanderbilt, Phys. Rev. B 56, R10024 (1999).

${ }^{3}$ T. Takeuchi, S. Sota, M. Katsuragawa, M. Komori, H. Takeuchi, H. Amano, and I. Akasaki, Jpn. J. Appl. Phys., Part 2 36, L382 (1997).

${ }^{4}$ P. Lefebvre, A. Morel, M. Gallart, T. Taliercio, J. Allègre, B. Gil, H. Mathieu, B. Damilano, N. Grandjean, and J. Massies, Appl. Phys. Lett. 78, 1252 (2001).

${ }^{5}$ S. Nakamura, Science 281, 956 (1998).

${ }^{6}$ K. P. O'Donnell, J. F. W. Mosselmans, R. W. Martin, S. Pereira, and M. E. White, J. Phys.: Condens. Matter 13, 6977 (2001).

${ }^{7}$ S. F. Chichibu, A. Uedono, T. Onuma, B. A. Haskell, A. Chakraborty, T. Koyama, P. T. Fini, S. Keller, S. P. Denbaars, J. S. Speck, U. K. Mishra, S. Nakamura, S. Yamaguchi, S. Kamiyama, H. Amano, I. Akasaki, J. Han, and T. Sota, Nat. Mater. 5, 810 (2006).

${ }^{8}$ A. Hangleiter, F. Hitzel, C. Netzel, D. Fuhrmann, U. Rossow, G. Ade, and P. Hinze, Phys. Rev. Lett. 95, 127402 (2005).

${ }^{9}$ A. Kaneta, K. Okamoto, Y. Kawakami, S. Fujita, G. Marutsuki, Y. Narukawa, and T. Mukai, Appl. Phys. Lett. 81, 4353 (2002).

${ }^{10}$ G. Franssen, S. Grzanka, R. Czernecki, T. Suski, L. Marona, T. Riemann, J. Christen, H. Teisseyre, P. Valvin, P. Lefebvre, P. Perlin, M. Lesczynski, and I. Grzegory, J. Appl. Phys. 97, 103507 (2005).

${ }^{11}$ F. Hitzel, G. Klewer, S. Lahmann, U. Rossow, and A. Hangleiter, Phys. Rev. B 72, 081309 (2005).

${ }^{12}$ S. Srinivasan, M. Stevens, F. A. Ponce, and T. Mukai, Appl. Phys. Lett. 87, 131911 (2005).

${ }^{13}$ S. Haffouz, H. Tang, J. A. Bardwell, P. Lefebvre, T. Bretagnon, T. Riemann, and J. Christen, J. Appl. Phys. 100, 013528 (2006).

${ }^{14}$ M. Merano, S. Sonderegger, A. Crottini, S. Collin, P. Renucci, E. Pelucchi, A. Malko, M. H. Baier, E. Kapon, B. Deveaud, and J.-D. Ganiére, Nature (London) 438, 479 (2005).

${ }^{15}$ M. Merano, S. Sonderegger, A. Crottini, S. Collin, E. Pelucchi, P. Renucci, A. Malko, M. H. Baier, E. Kapon, J.-D. Ganière, and B. Deveaud, Appl. Phys. B: Lasers Opt. 64, 343 (2006).

${ }^{16}$ S. M. Ting, J. C. Ramer, D. I. Florescu, V. N. Merai, B. E. Albert, A. Parekh, D. S. Lee, D. Lu, D. V. Christini, L. Liu, and E. A. Armour, J. Appl. Phys. 94, 1461 (2003).

${ }^{17}$ R. Aleksiejūnas, M. Sūdžius, V. Gudelis, T. Malinauskas, K. Jarašiūnas, Q. Fareed, R. Gaska, M. S. Shur, J. Zhang, J. Yang, E. Kuokštis, and M. Khan, Phys. Status Solidi C 7, 2686 (2003). 